\title{
ON A BLOCH-LANDAU CONSTANT
}

EDGAR REICH ${ }^{1}$

As usual, denote by $S$ the class of functions, $w=f(z)=z+a_{2} z^{2}$ $+\cdots$, regular and schlicht for $|z|<1$. Let $T$ be the subset of $S$ for which

$$
\left(1-|z|^{2}\right)\left|f^{\prime}(z)\right| \leqq 1,
$$$$
|z|<1
$$

Let $D_{f}$ denote the image of $\{|z|<1\}$ under $f(z)$.

THEOREM. $f \in T \rightarrow D_{f}$ contains the circle $\{|w|<.569\}$.

Proof. Inequality (1) implies (cf. [1])

$$
a_{2}=0, \quad\left|a_{3}\right| \leqq 1 / 3,
$$

and

$$
|f(z)| \leqq \frac{1}{2} \log \frac{1+|z|}{1-|z|}=|z| M(|z|) .
$$

For fixed $t, 0<t<1$, put $f(z, t)=f(t z) / t$. Thus

$$
|f(z, t)| \leqq M(t), \quad|z|<1 .
$$

Now let

$$
\tilde{f}(z, t)=M(t)\left[\phi\left\{\left(\frac{f(z, t)}{M(t)}\right)^{3}\right\}\right]^{1 / 3}=z+a_{3} t^{2} z^{3}+\cdots,
$$

where $\phi(z)=z /(1+z)^{2}$. If $f(z)$ omits $\gamma>0$, then $\tilde{f}(z, t)$ omits

$$
\gamma(t)=(\gamma / t)\left[1+\gamma^{3} / t^{3} M^{3}(t)\right]^{-2 / 3} .
$$

Let

$$
g(z, t)=\frac{\tilde{f}(z, t)}{(1-\tilde{f}(z, t) / \gamma(t))}=z+b_{2} z^{2}+b_{3} z^{3}+\cdots, \quad|z|<1 .
$$

We have

$$
b_{2}=1 / \gamma(t), \quad b_{3}=1 / \gamma^{2}(t)+a_{3} t^{2}, \quad\left|a_{3}\right| \leqq 1 / 3 .
$$

Now, since $g(z, t)$ is regular and schlicht for $|z|<1$, it follows $[2 ; 3]$ that

$$
\left|b_{3}-\alpha b_{2}^{2}\right| \leqq 2 e^{-2 \alpha /(1-\alpha)}+1 \text { for all } \alpha \in(0,1) .
$$

Received by the editors January 26, 1955 and, in revised form, March 16, 1955.

1 National Science Foundation post-doctoral fellow. 
This means

$$
|\gamma(t)| \geqq\left[\frac{3(1-\alpha)}{6 \exp \{-2 \alpha /(1-\alpha)\}+3+t^{2}}\right]^{1 / 2}, 0<\alpha<1 .
$$

In particular, when $\alpha=.372947, t=.99925$, a numerical computation shows that (4) and (5) jointly imply $|\gamma|>.569$.

CoRollary. $f \in S \rightarrow D_{f}$ contains a circle of radius $>.569$, i.e. the Bloch-Landau constant $\mathfrak{A}[1]$ satisfies the inequality $\mathfrak{A}>.569$.

Proof. As pointed out by Landau [1], it is sufficient to consider functions of class $T$ for the purpose of obtaining a lower bound on $\mathfrak{A}$.

The bound, .569 , obtained here is only very slightly better than

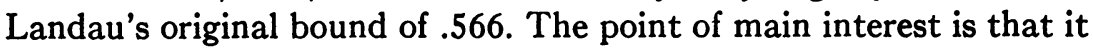
becomes clear that in order to improve Landau's bound one must make use of (1) globally for $|z|<1$, instead of just in the neighborhood of $z=0$, as Landau did.

\section{REFERENCES}

1. E. Landau, Über die Blochsche Konstante und zwei verwandte Weltkonstanten, Math. Zeit. vol. 30 (1929) pp. 608-634.

2. A. C. Schaeffer and D. C. Spencer, Coefficient regions for schlicht functions, Amer. Math. Soc. Colloquium Publications, vol. 35, 1950.

3. G. M. Goluzin, Some questions of the theory of univalent functions, Trudy Mat. Inst. Steklov. vol. 27 (1949) p. 46.

INSTITUTE FOR AdVANCEd STUdY 